Introduction à l'Electrodynamique Quantique Par Prof. Daniel Kastler. (Travaux et Recherches Mathematiques.) Pp. xxxiii + 333. (Paris: Dunod, 1961.) 68 N.F.

$\mathrm{T}$ HIS remarkable monograph has been written with the highly commendable aim of providing a common understanding between physicists and mathematicians, so far as the elements of quantum field theory are concerned. Whether this purpose has been achieved will probably be answered on the basis of the individual taste of any prospective reader. The present reviewer feels that the book will prove stimulating for the highly trained theoretical physicist, provided he has already acquired a fairly comprehensive familiarity with the customary formulation of quantum field theory.

The first two chapters and Appendixes $A, B, C$ provide the general mathematical tools and notions: vector-spaces, Hilbert space theory, the Lorentz group and its representations, tensor- and spinorcalculus. A short chapter on the method of second quantization precedes the classical and quantized theory of the scalar Klein-Gordon field, Maxwell field and Dirac field (Chapters 4-9). Chapter 10 is devoted to a general discussion of the coupled Maxwell and Dirac fields, and the next chapter surveys briefly the formalism of interacting fields in general, including the $S$-matrix theory. (The relationship between the $S$-matrix and scattering cross-sections is established in Appendix D.) Chapters 12-14 show, by examples of Compton scattering, Möller scattering and pair annihilation the applications of the theory. 'The Dyson-Feynman technique and diagrams are discussed only in the next chapter. The book concludes with a review of external field problems.

The mathematical exposition is 'high-brow' and follows the pattern of best French tradition; but most physicists will probably find it difficult to rediscover within this work the familiar physical concepts they are looking for. $\quad$ P. ROMAN

\section{Moon Atlas}

By V. A. Firsoff. Pp. $32+7$ plates +2 fold-out maps. (London: Hutchinson and Co. (Publishers), Ltd., 1961.) 63s. net.

THE descriptive classification of lunar surface features to be found at the beginning of this atlas is a commendably lucid account of the intricacies of lunar topography. Unfortunately, the illustrations appearing later in this atlas do little justice to the lunar photographs now available. The four quadrant maps of the lunar surface, although artistically drawn, have the annoying property of including very few names; identifications have to be made via co-ordinates of individual craters. Page 22 consists of a very handsome set of photographs of progressive lunar phases but of restricted value. The relief map of the Moon, extremely ingeniously drawn, is of value to young astronomers with small telescopes; it is doubtful, however, whether these persons will be able to afford the cost of the atlas. The spherical projection photographs add little value to the atlas: although there are few such photographs available elsewhere, Firsoff's efforts have not succeeded in producing pictures of sufficiently good quality to contribute much to selenological thought. There are good reproductions of the Full Moon, the Moon's averted hemisphere, and the well-known Palomar Observatory Clavius photograph. It is misleading that these latter should be included in the section on spherical projection photographs. The Moon Atlas closes with Firsoff's personal map of the lunar grid system. The presentation (in three colours) of this chart leaves something to be desired and I personally prefer to use the small-scale reproduction appearing on the back of the dust cover. BRIAN WARNER

\section{The International Dictionary of Physics and Elec- tronics}

Second edition. Pp. iv +1355. (Princeton, N.J.; D. Van Nostrand Company, Ltd., 1961.) 27.85 dollars; $205 s$.

THE standardization of terminology in physics and electronics is not an attractive task. Some national and international bodies have tackled parts of it with fair success, but because they seek agreement between organizations with widely differing experience and outlook, their progress is often slow. This comprehensive dictionary, the work of a handful of scientists with an overwhelmingly academic background, is therefore very welcome, even though one can find some unevenness and lack of balance-most likely attributable to the speed of compilation-and some failure to draw sufficiently directly on the works of the established standardization bodies.

Three features distinguish the second edition of the dietionary from the first, published in 1956. First, a new introduction shows how classical physics retreated before experimental findings of the early twentieth century and how new theories filled the gaps created; parts of the old introduction, on units and dimensions, are retained under appropriate headings in the body of the book. Secondly, the inclusion of many new items lengthens the book by about 200 pages and, thirdly, multilingual indexes (in French, German, Spanish and Russian) are added.

The title 'Dictionary' understates the value of the book, for some entries would fully satisfy, and many partially satisfy, the title 'Encyclopædia'; the book is therefore educational as well as one of reference. The next edition will have many new fields to cover, such as the properties of space, lasers and the applications of low-temperature physics; if it must accordingly increase in size, might not a shorter or concise edition, prepared by cutting the length rather than the number of entries, find a market as well ?

J. R. TILLMAN

The International Zoo Year Book

Vol. 1, 1959. Pp. vii + 160. 19 plates. Edited by Dr. Desmond Morris and Caroline Jarvis. (London: Zoological Society of London, 1960.) $40 \mathrm{~s}$.

TTHE International Zoo Year Book fulfils a very long-needed want, but $I$ feel it would be a great advantage if some check or verification could be made on the information given with regard to zoosparticularly in the Reference Section. To my know. ledge there are many inaccuracies, especially with regard to details concerning the smaller zoos. This is my only criticism-otherwise I think the book is a very worth-while effort which will prove of great benefit to all zoological gardens.

It is a great advantage to have a list of the senior staff of the zoos of the world, together with their addresses, and also the various departments of zoology. The London Zoological Society should be congratulated on the way this book has been produced. G. S. Motrershead 